\section{A incorporação do nusinersena no Sistema Único de Saúde: uma reflexão crítica sobre a institucionalização da avaliação de tecnologias em saúde no Brasil}

\author{
The incorporation of nusinersen by the Brazilian \\ Unified National Health System: critical thoughts \\ on the institutionalization of health technology \\ assessment in Brazil
}

\author{
La incorporación del nusinersén al Sistema \\ Único de Salud: una reflexión crítica sobre \\ la institucionalización de la evaluación de \\ tecnologías en salud en Brasil
}

Rosângela Caetano 1

Renata Curi Hauegen 2

Claudia Garcia Serpa Osorio-de-Castro 3

doi: 10.1590/0102-311X00099619

\section{Resumo}

Em abril de 2019, foi assinada a portaria de incorporação do medicamento nusinersena no Sistema Único de Saúde (SUS). É o medicamento mais caro já incorporado ao SUS, para uso no tratamento de atrofia muscular espinhal $5 q$ tipo I. A incorporação é referida como um marco na tomada de decisão sobre novas tecnologias no SUS, a ser viabilizada por meio de acordo de partilha de risco. O trabalho discute o processo de incorporação do nusinersena, destacando aspectos contextuais, temporais e técnicos, além de possíveis consequências para a institucionalização da avaliação de tecnologias em saúde (ATS) no SUS. Seguiu método exploratório, com revisão de informações públicas produzidas pela Comissão de Incorporação de Tecnologias no SUS (CONITEC) e busca em bancos de dados governamentais de preços e compras. Foi produzida linha temporal descrevendo os pontos-chave do processo de incorporação. Houve dois pedidos de incorporação do medicamento. O primeiro, submetido pela Secretaria de Ciência, Tecnologia e Insumos Estratégicos (SCTIE) do Ministério da Saúde, negado por unanimidade, em novembro de 2018. Seguiu-se o pedido do Secretário da SCTIE à Advocacia-Geral da União (AGU), para que pudesse decidir de forma contrária à recomendação do plenário da CONITEC. A AGU recomendou uma nova submissão, feita pela empresa produtora e aprovada por unanimidade, em março de 2019. Não houve acréscimo de novas evidências ou redução de preço que justificassem a mudança de decisão. Não foram identificados os elementos constituintes do acordo de partilha de risco. São sinalizados problemas de transparência e accountability, bem como riscos ao processo de institucionalização da ATS que vinha em curso no SUS.

Avaliação de Tecnologias em Saúde; Medicamento Órfão; Participação no Risco Financeiro; Sistema Único de Saúde

\section{Correspondência}

R. Caetano

Departamento de Planejamento e Administração em Saúde, Instituto de Medicina Social, Universidade do Estado do Rio de Janeiro.

Rua São Francisco Xavier 524, 7o andar, Blocos D/E, Rio de Janeiro, RJ 20559-900, Brasil.

caetano.r@gmail.com

1 Instituto de Medicina Social, Universidade do Estado do Rio de Janeiro, Rio de Janeiro, Brasil.

2 Centro de Desenvolvimento Tecnológico em Saúde,

Fundação Oswaldo Cruz, Rio de Janeiro, Brasil.

3 Escola Nacional de Saúde Pública Sergio Arouca,

Fundação Oswaldo Cruz, Rio de Janeiro, Brasil. 


\section{Introdução}

Em abril de 2019, a assinatura da Portaria de incorporação do medicamento nusinersena no Sistema Único de Saúde (SUS) mereceu sessão solene no Senado Federal, ganhando destaque na mídia e nas páginas governamentais. Essa incorporação é referida como um marco no tratamento das doenças raras, a ser viabilizada com uma nova modalidade de aquisição, a partilha de riscos. Por intermédio desse modelo contratual, Estado e fornecedor concordam que a importância a ser paga pela tecnologia dependerá das evidências de mundo real, coletadas com base na prática clínica 1.

O nusinersena (Spinraza, Biogen Brasil Produtos Farmacêuticos Ltda.) é destinado ao tratamento da atrofia muscular espinhal (AME), doença neuromuscular de origem genética recessiva rara, com incidência de 1:10.000 nascidos vivos e prevalência estimada de 1-2:100.000 pessoas 2. Causada por mutações do gene SMN1 no cromossoma 5, a AME é caracterizada pela degeneração dos neurônios motores da medula espinhal e tronco encefálico, conduzindo à atrofia e paralisia progressivas e necessidade de suporte ventilatório, nas suas formas mais graves ${ }^{3}$. Tem cinco fenótipos clínicos - 0, I, II, III e IV - em termos de seu início de apresentação e gravidade. O tipo I, para o qual houve incorporação no SUS, corresponde a cerca de $60 \%$ dos casos, com manifestação precoce dos sintomas, usualmente nos primeiros seis meses e elevada mortalidade até dois anos de idade 4 . A posologia recomendada para qualquer idade, área ou peso corporal corresponde a $12 \mathrm{mg}$ por via intratecal (seis doses no primeiro ano e três doses de manutenção subsequente), com critérios também definidos para a descontinuação da terapêutica.

É um medicamento registrado no país em 2017 5. Seu custo é muito elevado e, até abril de 2019, as compras decorriam de judicialização dos entes governamentais.

Desde a Lei no 12.410/2011 6, a avaliação para a incorporação de tecnologias no SUS é realizada pela Comissão Nacional de Incorporação de Tecnologias (CONITEC), que tem a função regimental de assessorar o Ministério da Saúde nestas decisões. Essa avaliação deve seguir critérios definidos em lei, sendo exigidos, dentre outros, registro válido na Agência Nacional de Vigilância Sanitária (Anvisa), apresentação de evidências científicas de eficácia, segurança e custo-efetividade, exame do impacto orçamentário e preço fixado pela Câmara de Regulação do Mercado de Medicamentos (CMED) da Anvisa, no caso de medicamentos. Uma recomendação preliminar é submetida à consulta pública, embora o regimento legal - Decreto no 7.646/2011 7 - preveja situações onde esta seja substituída por um processo administrativo simplificado. Após a apreciação das contribuições, a recomendação final do plenário é submetida ao secretário da Secretária de Ciência, Tecnologia e Insumos Estratégicos do Ministério da Saúde (SCTIE/MS) para a decisão 6,7.

O estudo discute o processo de incorporação do nusinersena, destacando aspectos contextuais, temporais e técnicos, além de possíveis consequências para a institucionalização da avaliação de tecnologias no SUS.

\section{Métodos}

Estudo exploratório, de caráter retrospectivo, que utilizou apenas dados de fontes públicas.

Extraiu-se do Sistema Integrado de Administração de Serviços Gerais (SIASG) o número de compras, quantidades adquiridas, preços unitários praticados e valores dos gastos contratados referentes às compras de nusinersena no período de janeiro de 2017 a abril de 2019, com vistas a consolidar os gastos federais com o medicamento até sua incorporação pelo SUS.

Os preços máximos de venda ao governo (PMVG), desde o registro sanitário do medicamento no país, foram obtidos nas tabelas mensais disponibilizadas pela CMED (http://portal.anvisa.gov.br/ listas-de-precos).

Os registros das demandas de incorporação, relatórios técnicos de recomendação e contribuições às consultas públicas foram levantados no website da CONITEC (http://conitec.gov.br). Adicionalmente, realizou-se busca por literatura cinzenta nas páginas eletrônicas do Executivo e Legislativo federais. Usando-se análise temática dos conteúdos, buscamos mapear a evolução temporal e justificativas relacionadas ao processo de incorporação. Foi elaborada uma linha de tempo contendo os principais eventos deste processo. 
Para estabelecer comparação quantitativa, foram contabilizadas todas as contribuições de todas as consultas públicas relativas aos relatórios com parecer favorável de incorporação pela CONITEC de janeiro de 2012 a abril de 2019.

\section{Resultados}

O registro do nusinersena no Brasil foi obtido em agosto de 2017, para as populações pediátrica e adulta e todos os tipos de AME 5. Compras desse medicamento estão registradas no SIASG desde janeiro de 2018 (Tabela 1), todas para atendimento a demandas judiciais. Em 2018, foram contratadas 69 compras, para a aquisição de 496 unidades; em 2019, até 28 de abril, oito compras, correspondentes a 118 unidades. Ao final dos 16 meses, em exame foram gastos pelo Governo Federal cerca de R\$ 143 milhões, para o suprimento de 614 frascos do medicamento, o que corresponderia ao tratamento inicial de 102 pacientes, caso estivessem todos no primeiro ano de uso. Há uma tendência de aumento de unidades compradas e de diminuição no número de compras.

O primeiro PMVG foi atribuído na tabela CMED de novembro de 2017 8. Em abril de 2018, foi majorado em 2,09\% 9 . Em outubro de 2018, ocorreu redução de 1,09\% no PMVG 10, que vigorou inalterado até março de 2019. Em abril de 2019, o PMVG com ICMS de 0\% foi de R\$247.399,94 (+4,33\%), e não há registro de valor para PMVG sem impostos 11. Em maio de 2019, o PMVG com ICMS de 0\% se manteve inalterado, e aparece valor de PMVG sem impostos de R\$ 220.892,77 12 (Tabela 2). O preço médio mensal pago nas compras governamentais em 2018 foi de R\$238.576,25, caindo a R\$ 209.117,13, em 2019, antes mesmo da incorporação (Tabela 1).

\section{Tabela 1}

Número de compras federais e de unidades adquiridas, gastos contratados e preço médio ponderado (PMP) mensal do nusinersena, de janeiro de 2018 a abril de 2019.

\begin{tabular}{|c|c|c|c|c|}
\hline Ano/Mês & Compras & $\begin{array}{l}\text { Unidades } \\
\text { adquiridas }\end{array}$ & $\begin{array}{c}\text { Gasto contratado } \\
\text { (R\$ correntes) }\end{array}$ & $\begin{array}{l}\text { PMP mensal } \\
\text { (R\$ correntes) }\end{array}$ \\
\hline \multicolumn{5}{|l|}{2018} \\
\hline Janeiro & 2 & 12 & $2.798 .822,04$ & $233.235,17$ \\
\hline Fevereiro & 2 & 12 & $3.586 .249,44$ & $298.854,12$ \\
\hline Março & 10 & 56 & $13.698 .773,88$ & $244.620,96$ \\
\hline Abril & 10 & 57 & $14.377 .276,50$ & $252.232,92$ \\
\hline Maio & 2 & 12 & $3.025 .978,08$ & $252.164,84$ \\
\hline Junho & 8 & 48 & $12.320 .974,14$ & $256.686,96$ \\
\hline Julho & 7 & 38 & $9.570 .123,19$ & $251.845,35$ \\
\hline Agosto & 5 & 78 & 19.819.377,12 & $254.094,58$ \\
\hline Setembro & 0 & 0 & - & - \\
\hline Outubro & 11 & 71 & $15.764 .515,27$ & $222.035,43$ \\
\hline Novembro & 3 & 67 & $13.981 .309,89$ & $208.676,27$ \\
\hline Dezembro & 9 & 45 & $9.390 .421,95$ & $208.676,04$ \\
\hline Subtotal & 69 & 496 & $118.333 .821,50$ & $238.576,25$ \\
\hline \multicolumn{5}{|l|}{2019} \\
\hline Janeiro & 7 & 35 & $7.355 .692,75$ & $210.162,65$ \\
\hline Fevereiro & 0 & 0 & - & - \\
\hline Março & 0 & 0 & - & - \\
\hline Abril & 1 & 83 & $17.320 .128,75$ & $208.676,25$ \\
\hline Subtotal & 8 & 118 & $24.675 .821,50$ & $209.117,13$ \\
\hline Total & 77 & 614 & $143.009 .643,00$ & $232.914,73$ \\
\hline
\end{tabular}

Fonte: Sistema Integrado de Administração de Serviços Gerais (SIASG), 2018-2019. 


\section{Tabela 2}

Preço máximo de venda ao governo (PMVG) do medicamento nusinersena (frasco ampola $5 \mathrm{~mL}, 2,4 \mathrm{mg} / \mathrm{mL}$ ) disposto nas tabelas mensais da Câmara de Regulação do Mercado de Medicamentos (CMED), Agência Nacional de Vigilância Sanitária (Anvisa), 2017-2019.

\begin{tabular}{|c|c|c|}
\hline Ano/Mês & PMVG com ICMS 0\% & PMVG com ICMS $20 \%$ \\
\hline \multicolumn{3}{|l|}{2017} \\
\hline Agosto * & - & - \\
\hline Setembro & - & - \\
\hline Outubro & - & - \\
\hline Novembro ** & $234.837,73$ & $302.797,58$ \\
\hline Dezembro & $234.837,73$ & $302.797,58$ \\
\hline \multicolumn{3}{|l|}{2018} \\
\hline Janeiro & $234.837,73$ & $302.797,58$ \\
\hline Fevereiro & $234.837,73$ & $302.797,58$ \\
\hline Março & $234.837,73$ & $302.797,58$ \\
\hline Abril *** & $239.745,83$ & $309.126,05$ \\
\hline Maio & $239.745,83$ & $309.126,05$ \\
\hline Junho & $239.745,83$ & $309.126,05$ \\
\hline Julho & $239.745,83$ & $309.126,05$ \\
\hline Agosto & $239.745,83$ & $309.126,05$ \\
\hline Setembro & $239.745,83$ & $309.126,05$ \\
\hline Outubro \# & $237.132,15$ & $305.756,00$ \\
\hline Novembro & $237.132,15$ & $305.756,00$ \\
\hline Dezembro & $237.132,15$ & $305.756,00$ \\
\hline \multicolumn{3}{|l|}{2019} \\
\hline Janeiro & $237.132,15$ & $305.756,00$ \\
\hline Fevereiro & $237.132,15$ & $305.756,00$ \\
\hline Março & $237.132,15$ & $305.756,00$ \\
\hline Abril *** & 247.399,94 \#\# & $318.995,23$ \\
\hline Maio & 247.399,94 \#\#\# & $318.995,23$ \\
\hline
\end{tabular}

ICMS: Imposto sobre Circulação de Mercadorias e Serviços.

* Mês de registro do medicamento pela Anvisa;

** Mês de primeira precificação pela CMED;

*** Mês de reajuste anual de preços de medicamentos no país;

\# Alteração fora do período usual de reajustes de preços de medicamentos;

\#\# Não existe PMVG sem impostos presente na tabela CMED (valor registrado = 0);

\#\#\# PMVG sem impostos estabelecidos como R\$220.892,77.

Os principais eventos relacionados à incorporação do nusinersena estão sumarizados no Quadro 1, e o Quadro 2 elenca as principais recomendações relativas ao medicamento ao longo do processo.

Ocorreram duas solicitações de apreciação de incorporação do medicamento, separadas por um ano. A primeira demanda foi submetida pela SCTIE/MS em janeiro de 2018. Recomendação preliminar de não incorporação foi feita na 69a reunião ordinária de agosto de 201813 e submetida à consulta pública por 20 dias, tendo recebido 36.972 contribuições 14 . Na 72 a reunião ordinária, em novembro de 2018, as contribuições foram apreciadas 15 . Conforme ata pública dessa reunião, estudos publicados após a busca feita para o relatório da CONITEC foram revisados e seus resultados corroboravam a recomendação de não incorporação 15 . O Plenário entendeu que não houve evidência suficiente para alterar sua recomendação inicial e deliberou, por unanimidade, manter a não incorporação 15 . O Relatório no 400 correspondente à deliberação final não está disponível nas páginas eletrônicas da CONITEC; a demanda consta apenas como "processo encerrado a pedido do demandante" 16. 


\section{Quadro 1}

Principais eventos relacionados à incorporação do nusinersena para a atrofia muscular espinhal $5 q$ tipo I no Sistema Único de Saúde.

\begin{tabular}{|c|c|c|}
\hline & DATA & EVENTO \\
\hline 1 & 28/Agosto/2017 & Registro do nusinersena pela Anvisa. \\
\hline 2 & 27/Novembro/2017 & Primeira atribuição de PMVG do nusinersena nas tabelas CMED. \\
\hline 3 & 23/Janeiro/2018 & Protocolada demanda de avaliação pela CONITEC originária da SCTIE/MS. \\
\hline 4 & 02/Agosto/2018 & $\begin{array}{l}\text { 69ạ Reunião Ordinária da CONITEC - recomendação preliminar de não incorporação para AME 5q tipo I. } \\
\text { Relatório preliminar CONITEC (sem nô), de agosto/2018, para consulta pública. } \\
\text { Relatório para a Sociedade CONITEC no 111, de agosto/2018. }\end{array}$ \\
\hline 5 & $\begin{array}{l}\text { 29/Agosto a } 17 / \\
\text { Setembro/2018 }\end{array}$ & Consulta Pública no 45, de 2018. \\
\hline 6 & 08/Novembro/2018 & 72ạ Reunião Ordinária da CONITEC - recomendação final de não incorporação para AME 5q tipo I. \\
\hline 7 & Dezembro/2018 & $\begin{array}{l}\text { Despacho SCTIE/MS s/n (documento SEI “7088380”) solicitando posicionamento da AGU e dando origem ao } \\
\text { processo } 25000.013521 / 2018-95 \text {. }\end{array}$ \\
\hline 8 & 18/Dezembro/2018 & $\begin{array}{l}\text { Parecer AGU no 01377/2018/CONJUR-MS/CGU/AGU, recomendando que a SCTIE/MS, caso decida por não } \\
\text { adotar a recomendação da CONITEC, submeta o caso à nova avaliação da Comissão. }\end{array}$ \\
\hline 9 & 22/Janeiro/2019 & Protocolada demanda de avaliação pela CONITEC originária da Biogen Brasil Produtos Farmacêuticos Ltda. \\
\hline 10 & 14/Março/2019 & $\begin{array}{l}\text { 75ạ Reunião Ordinária da CONITEC - recomendação preliminar de incorporação para AME 5q tipo I. } \\
\text { Relatório preliminar CONITEC (sem no), de março/2019, para consulta pública. } \\
\text { Relatório para a Sociedade CONITEC no 145, de março/2019. }\end{array}$ \\
\hline 10 & 19/Março a 28/Março/2019 & Consulta Pública no 12, de 2019. \\
\hline 11 & 04/Abril/2019 & 76ạ Reunião Ordinária da CONITEC - recomendação final de incorporação para AME 5q tipo I. \\
\hline 12 & 24/Abril/2019 & $\begin{array}{l}\text { Portaria SCTIE no } 24 / 2019 \text { - decisão de incorporação para AME 5q tipo I. } \\
\text { Relatório CONITEC de recomendação no 449/2019. }\end{array}$ \\
\hline
\end{tabular}

AGU: Advocacia Geral da União; AME: atrofia muscular espinhal; Anvisa: Agência Nacional de Vigilância Sanitária; CMED: Câmara de Regulação do Mercado de Medicamentos/Anvisa; CONITEC: Comissão Nacional de Incorporação de Tecnologias no SUS; PMVG: preço máximo de venda ao governo; SCTIE/MS: Secretaria de Ciência, Tecnologia e Insumos Estratégicos/Ministério da Saúde.

Em dezembro de 2018, a Advocacia Geral da União (AGU) emitiu o Parecer no 01377/2018/ CONJUR-MS/CGU/AGU $17 \mathrm{em}$ atendimento à consulta do então Secretário da SCTIE/MS, que versava sobre a possibilidade de sua decisão em sentido diverso da recomendação do Plenário da CONITEC. Segundo o Parecer, o Despacho SCTIE s/n (documento SEI “7088380") traz como "principal motivação do não acatamento do relatório da CONITEC a existência de uma nova proposta de preço por parte da empresa Biogen, proposta essa ocorrida após a avaliação já realizada pela CONITEC” (argumento 38). Afirma que, ao entender como "pertinente a incorporação do medicamento nusinersena para todos os com AME 5q, independentemente de fenótipo..." (p. 3-4), o Despacho realiza "ampliação objetiva do escopo técnico da matéria”, uma vez que a apreciação da CONITEC se limitou à indicação para AME 5q tipo I. A AGU discute também que a nova proposta de preço não resolve a "fragilidade das evidências clínicas referidas na recomendação da CONITEC" (argumento 31) 17.

O Parecer transcreve o resumo executivo do Relatório no 400 da CONITEC, destacando os critérios que subsidiaram a recomendação de não incorporação (argumento 36). Diante das atribuições legais de competência, ressalta que, apesar do caráter de assessoramento das atividades da CONITEC, "não se pode concluir pela existência de um poder amplo e ilimitado de revisão, pelo Secretário da SCTIE, do entendimento exarado pela CONITEC" (argumento 23). Finaliza, considerando que "não obstante haver, de fato, a possibilidade do Secretário da SCTIE/MS, a priori, decidir", pode "haver grave insegurança jurídica na opção pela decisão de não acatamento da recomendação da CONITEC”. Orienta que a SCTIE/MS, caso opte por não acatar a recomendação da CONITEC, promova nova submissão 17. 


\section{Quadro 2}

Comparação das recomendações presentes nos documentos da Comissão Nacional de Incorporação de Tecnologias no SUS (CONITEC) relacionados à incorporação do nusinersena no Sistema Único de Saúde (SUS).

\begin{tabular}{|c|c|c|c|c|}
\hline $\begin{array}{l}\text { Relatório preliminar } \\
\text { enviado à Consulta } \\
\text { Pública } n^{\circ} 45 \\
\text { (agosto/2018) }\end{array}$ & $\begin{array}{c}\text { Relatório } n^{\circ} 400 \\
\text { (dezembro/2018) * }\end{array}$ & $\begin{array}{l}\text { Relatório preliminar } \\
\text { enviado à Consulta } \\
\text { Pública } n^{\circ} 12 \\
\text { (março/2019) }\end{array}$ & $\begin{array}{c}\text { Relatório } n^{\circ} 449 \\
\text { (abril/2019) }\end{array}$ & Portaria n 24 (abril/2019) \\
\hline 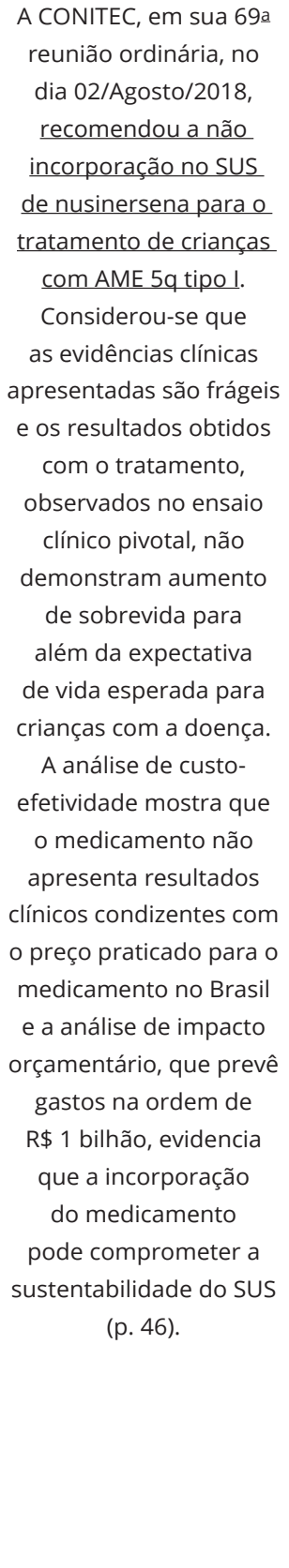 & 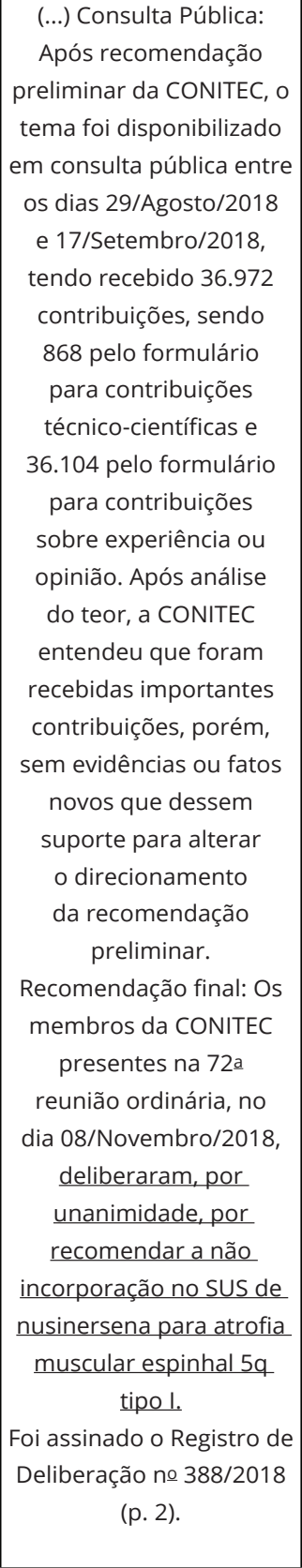 & 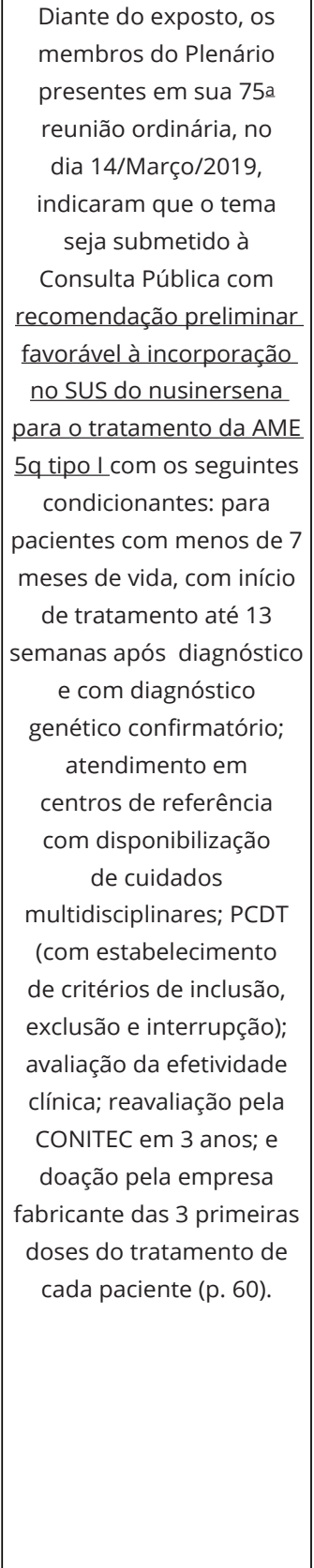 & 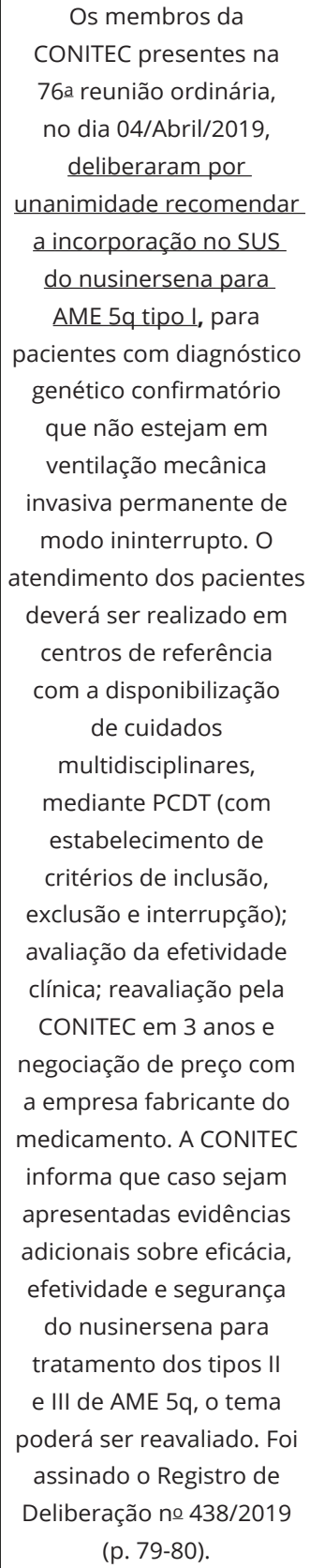 & 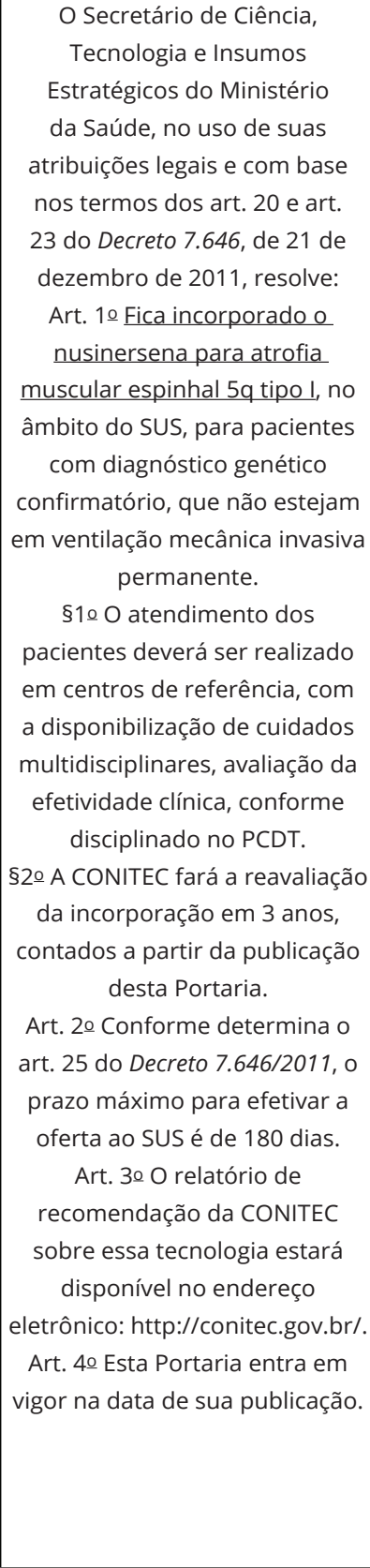 \\
\hline
\end{tabular}

AME: atrofia muscular espinhal; PCDT: protocolo clínico e diretrizes terapêuticas.

* O relatório final de no 400 não está nas páginas eletrônicas da CONITEC, onde a informação disponível é que a demanda proveniente da Secretária de Ciência, Tecnologia e Insumos Estratégicos do Ministério da Saúde foi retirada a pedido do demandante. A recomendação transcrita é procedente do parecer da Advocacia Geral da União no 01377/2018/CONJUR-MS/CGU/AGU, que transcreve o sumário executivo do referido documento. 
Em janeiro de 2019, nova submissão foi realizada, com indicação sem restrição por tipo de AME, tendo por demandante o fabricante. No relatório preliminar da CONITEC de março de 2019 consta, contudo, recomendação favorável de incorporação apenas para o tratamento da AME 5q tipo I, por considerar que os estudos avaliando a doença de início tardio eram escassos 18 . O preço proposto pelo fabricante é relatado como de R $145.700,00$ por frasco. Incluíram avaliação econômica tendo como comparador o não uso de tratamento modificador da doença, que apresentou razão de custo-efetividade incremental (RCEI) para a AME de início precoce de $\mathrm{R} \$ 749.276,00$ a $\mathrm{R} \$ 1.023 .351,00$ por ano de vida ganho ajustado por qualidade (QALY), dependendo de quem avaliava as utilidades. O documento elenca ainda condicionantes para a incorporação: exigência de diagnóstico genético confirmatório; restrição a pacientes menores de 7 meses de vida e com início de tratamento até 13 semanas após o diagnóstico; e doação pelo fabricante das três primeiras doses do tratamento 18.

A nova recomendação foi submetida à consulta pública por 10 dias, recebendo 41.787 contribuições. Dos 172 relatórios com recomendação final favorável de incorporação pela CONITEC entre janeiro de 2012 e abril de 2019, 123 foram submetidos à consulta pública. As contribuições do nusinersena correspondem a 55,8\% do total de 74.900 contribuições (Tabela 3). O número máximo de contribuições até então tinha ocorrido na avaliação das insulinas análogas de ação prolongada para o tratamento de diabetes mellitus tipo I (2.574).

Em 4 de abril, apenas uma semana após o término da consulta, o Plenário da Comissão deliberou, também por unanimidade, pela incorporação do fármaco para o tratamento da AME 5q tipo I 18.

No relatório final da CONITEC, as condições estabelecidas diferem da recomendação preliminar. Continua exigido o diagnóstico confirmatório e que os pacientes não estejam em ventilação mecânica invasiva permanente contínua, mas não existem menções à idade ou ao intervalo de tempo para o início do tratamento. Também não há mais referência à doação de frascos e sim à negociação de preço com o fabricante. Consta ainda que, caso sejam apresentadas evidências adicionais sobre eficácia, efetividade e segurança do nusinersena para o tratamento dos tipos II e III de AME 5q, o tema poderá ser reavaliado pela CONITEC 19.

Nos relatórios preliminar e final, existe a recomendação que os pacientes atendidos sejam acompanhados em centros de referência, com base em protocolo clínico e diretrizes terapêuticas (PCDT) com critérios de inclusão, exclusão e interrupção estabelecidos; coleta de dados em sistema informatizado que permita a avaliação da efetividade clínica; e reavaliação pela CONITEC em três anos 18,19.

\section{Tabela 3}

Número de contribuições à Consulta Pública nos relatórios que receberam recomendação favorável de incorporação pelo plenário da Comissão de Incorporação de Tecnologias no SUS (CONITEC), janeiro de 2012 a abril de 2019.

\begin{tabular}{|c|c|c|c|c|c|c|c|}
\hline \multirow[t]{2}{*}{ Ano } & \multicolumn{2}{|c|}{ Recomendações favoráveis } & \multirow[b]{2}{*}{ Total no ano } & \multicolumn{3}{|c|}{ Contribuições } & \multirow[b]{2}{*}{$\begin{array}{c}>1.000 \\
\text { contribuições }\end{array}$} \\
\hline & $\mathbf{n}$ & $\begin{array}{c}\% \text { com Consulta } \\
\text { Pública }\end{array}$ & & $\begin{array}{l}\text { Menor/Maior } \\
\text { número }\end{array}$ & Média & $\begin{array}{c}>500 \\
\text { contribuições }\end{array}$ & \\
\hline 2012 & 15 & 60 & 618 & $0 / 262$ & 68,7 & 0 & 0 \\
\hline 2013 & 24 & 83,3 & 349 & $0 / 130$ & 17,5 & 0 & 0 \\
\hline 2014 & 26 & 53,8 & 685 & $0 / 530$ & 48,9 & 1 & 0 \\
\hline 2015 & 22 & 36,4 & 1.398 & $4 / 392$ & 174,5 & 0 & 0 \\
\hline 2016 & 13 & 76,9 & 796 & $4 / 284$ & 79,6 & 0 & 0 \\
\hline 2017 & 27 & 74,1 & 7.388 & $5 / 1.813$ & 369,4 & 6 & 2 \\
\hline 2018 & 35 & 94,3 & 18.521 & $1 / 2.444$ & 561,2 & 13 & 7 \\
\hline 2019 * & 10 & 90 & 45.145 & $19 / 41.787 * *$ & $5.016,10$ & 2 & 2 \\
\hline Total & 172 & 71,5 & 74.900 & $0 / 41.787$ & NA & 22 & 11 \\
\hline
\end{tabular}

NA: não aferido.

Fonte: CONITEC (Recomendações da CONITEC, última atualização em 25/Abr/2019. http://conitec.gov.br/decisoes-sobre-incorporacoes; acessado em 07/Mai/2019).

* Recomendações realizadas até abril de 2019;

** Número de contribuições do nusinersena. 
A Portaria SCTIE no 24 foi assinada em 24 de abril de 2019 20. A sessão ocorreu no Senado, com o Ministro da Saúde informando que a aquisição do nusinersena seria realizada sob nova modalidade de compras, a partilha de risco.

\section{Discussão}

O registro do nusinersena no Brasil aconteceu depois de rápida sucessão em várias agências no mundo. Ao contrário de muitos medicamentos de alto custo judicializados e adquiridos sem registro 21 , o nusinersena teve registro deferido antes que os gastos com atendimento de demandas judiciais fossem constatados nas compras federais. Isso pode ser explicado pelo julgamento do Tema 106 de repercussão geral no Supremo Tribunal de Justiça (STJ) 22, que obriga o registro na Anvisa para a concessão de medicamentos não incorporados ao SUS. As ações podem ter sido ajuizadas antes, mas as primeiras compras contratadas aparecem em janeiro de 2018.

De janeiro a dezembro de 2018, apenas com compras de 496 frascos de nusinersena, os gastos federais somaram R\$ 118 milhões, em comparação a gastos de R\$ 447,16 milhões dispendidos com as aquisições de 32.877 unidades do eculizumabe, em ambos os casos sempre para atendimento de demanda judicial.

Trata-se do medicamento mais caro já adquirido pelo sistema público brasileiro, com seu PMVG chegando a R\$247.399,94 por frasco. Contudo, ainda que o PMVG represente o teto de preço para a compra de qualquer medicamento adquirido por força de decisão judicial, os preços praticados em todas as aquisições ocorridas de fevereiro a agosto de 2018 estiveram acima do estabelecido, como pode ser visto na Tabela 1.

O medicamento já teve seu preço majorado duas vezes, em 2,09\% e 4,33\%, respectivamente, em abril de 2018 e 2019, à época do reajuste anual de preços de medicamentos no Brasil. Além disso, sofreu uma redução de preço de tabela $(-1,09 \%)$ em outubro de 2018 , evidenciando flutuações de preço mesmo com tão pouco tempo no mercado.

Análises de incorporação preocupam-se primordialmente com segurança, eficácia e efetividade da tecnologia em condições reais de uso 6, uma vez que pressupõem escolha de financiamento sobre outras tecnologias em competição. Medicamentos que podem comprometer a sustentabilidade do SUS devem requerer evidências robustas para a incorporação. No Brasil, um estudo aponta para a rejeição de tecnologias que impliquem, com sua entrada, a eliminação de outras ou de programas, ou carga adicional para a sociedade 23 .

A segunda submissão de avaliação, realizada um mês após o parecer da AGU, atende a orientação da necessidade de nova apreciação pela CONITEC, evitando a situação qualificada como de "grave insegurança jurídica” na hipótese de decisão monocrática do secretário da SCTIE/MS distinta da recomendação da CONITEC. Ressalta-se, contudo, que a nova submissão foi realizada pela indústria produtora e não pela SCTIE/MS.

Ainda que a Lei preveja a possibilidade do secretário da SCTIE decidir em desacordo com a Comissão, o poder discricionário atribuído ao ato requer motivos relevantes a justificá-lo. Os aspectos destacados no parecer da AGU diziam respeito a "fato novo" (proposta de redução do preço) e à ampliação pretendida do escopo inicial, não presentes no momento da avaliação em 2018. Esses aspectos deveriam, pois, ser objeto da nova apreciação, usando-se os mesmos critérios técnicos e econômicos que governam toda e qualquer incorporação ao SUS.

O relatório preliminar de março de 201918 recomendava, como um dos condicionantes para a incorporação, a doação de três frascos por paciente, e as avaliações econômicas e de impacto orçamentário foram conduzidas tomando-se por base um preço de R\$ 145 mil por frasco. Nesse sentido, é preciso refletir sobre a evolução dos acontecimentos após a segunda consulta. Nela, a Biogen afirma que "...não foi consultada e tem importantes questionamentos relacionados ao modelo sugerido pelo relatório da CONITEC (fornecimento de três frascos sem custo). Entendemos que esse modelo não faz sentido frente a indicação restritiva que a recomendação apresenta (...). Sobre o preço proposto para incorporação, cabe ratificar que o mesmo foi ofertado mediante a incorporação de nusinersena para todos os pacientes com AME $5 q$ (...). Qualquer recomendação que restrinja o acesso ou não contemple a indicação de bula do medicamento, conforme aprovado pela ANVISA, requererá nova negociação” 24 (p. 20-1). 
Naquele momento, não havia evidências suficientes que dessem suporte à recomendação de incorporação para os tipos II e III. O próprio relatório final destaca que a CONITEC poderia reavaliar o assunto caso fossem "....apresentadas evidências adicionais sobre eficácia, efetividade e segurança do nusinersena para tratamento dos tipos II e III de AME 5q" 19 (p. 80). Portanto, ao final da Consulta Pública que incluiu a contribuição da Biogen 24, o plenário da CONITEC e a SCTIE/MS sabiam que a alteração no preço, suposto "fato novo" que justificaria a nova avaliação segundo a AGU, não se confirmaria. A decisão foi tomada mesmo com expressa negativa da empresa em assumir a proposta de R $\$ 145 \mathrm{mil} /$ frasco 24. A indicação aprovada no SUS não atendeu ao pretendido pelo fabricante e o preço de venda proposto no final do processo não demonstrou qualquer economia.

Na tabela CMED de maio de 2019, publicada após a incorporação, o PMVG com ICMS 0\% persiste como em abril em R \$ 247.399,94, mas há registro de PMVG sem impostos de R\$220.892,77, representando uma redução de 10,7\% 12. Ressalta-se, contudo, que na compra feita pelo Ministério da Saúde em abril de 2019 (83 unidades) o preço de aquisição do nusinersena já era de R $\$ 208.276,25$, inferior a esse preço sem impostos.

A empresa negou a proposta de doação de três frascos. O relatório não apresenta qualquer justificativa para tal número, que sequer atinge a dose de ataque no primeiro ano de tratamento (seis frascos, independentemente do peso corporal do paciente). No relatório final, a questão da doação desaparece e a condicionalidade à negociação de preço com o fabricante passa a estar presente.

Destaca-se que a incorporação não se limita a um julgamento financeiro e é preciso considerar, antes de tudo, os benefícios e as evidências. A economia para o SUS estaria diretamente condicionada não à diminuição de preço, per se, mas à demonstração de novos benefícios da terapêutica. Caso contrário, a incorporação não se justificaria. Para entender a decisão de incorporação do nusinersena, tomada em detrimento aparente de diversos condicionantes que apontariam para outro curso, é preciso verificar que outros fatos novos, para além dos citados no parecer da AGU, existiriam para garantir que um novo exame encontraria uma relação de benefício diferente da avaliação anterior. $O$ Relatório no 400 da CONITEC, citado pela AGU, menciona "fragilidades nas evidências" 17.

No caso da AME 5q tipo I, os relatórios da CONITEC de agosto de 2018 e de março de 2019 diferem pouco em relação às evidências utilizadas, mencionando um ensaio clínico controlado randomizado (ECCR), um ensaio controlado aberto e três coortes de seguimento 13,17. Três estudos prospectivos só foram incluídos no último relatório 18 . A avaliação da qualidade metodológica é também muito semelhante nos dois documentos, com o ensaio aberto e duas coortes sem poderem ser avaliadas por ser tratarem de resumos de congresso, a qualidade do ECCR considerada moderada e a das demais coortes, baixa.

A principal peça de evidência é o ECCR fase III ENDEAR, publicado em novembro de 201725. Os pacientes do grupo intervenção mostraram maior probabilidade de sobrevida livre de eventos (tempo até morte ou entrada em ventilação assistida permanente por $\geq 16$ horas por mais de 21 dias contínuos) e de sobrevida global que os do grupo controle, apenas para pacientes que iniciaram o tratamento até 13,1 semanas após o diagnóstico. O grupo de intervenção também apresentou melhora de "marcos motores", desfecho dicotômico construído com base em escores obtidos da escala HINE-2. Contudo, existem controvérsias quanto ao desfecho combinado utilizado, pela sua característica extremamente geral (aceitando como resultados de igual importância desde movimentos leves de cabeça e pescoço até o ficar em pé e o deambular) e por sua não validação 26 .

Nenhuma nova evidência foi apresentada que justificasse a mudança de recomendação. $O$ fato de um único ensaio fase III de qualidade metodológica moderada subsidiar a incorporação de um medicamento de altíssimo custo para o sistema, com impacto orçamentário total, acumulado em cinco anos, de R\$1,2 a mais de R \$ 1,6 bilhão dependendo da taxa de difusão da tecnologia, sugere a pergunta: as evidências produzidas e apresentadas são suficientes?

O Brasil não tem um limiar de custo-efetividade estabelecido que defina o que seria uso eficiente dos recursos e determine valor abaixo do qual se possa justificar a incorporação de uma tecnologia 27. O estabelecimento e o uso desse limiar envolvem dificuldades e controvérsias de várias ordens 28 , inclusive sobre sua aplicação para doenças raras 29 . Uma discussão mais aprofundada dessas questões foge ao escopo deste estudo, mas não se questiona que os valores da RCEI estimados são bastante elevados. 
Questiona-se também por que a recomendação final de abril de 2019 não traz mais como condicionante as restrições quanto à idade do paciente ( $<7$ meses de vida) e ao início de tratamento (até 13 semanas após o diagnóstico) ${ }^{19}$. Essa eliminação no texto final permite, na prática, uma ampliação da população-alvo candidata à terapêutica. Por outro lado, dada a exigência de diagnóstico genético confirmatório, dificuldades no acesso ao diagnóstico podem retardar o uso. Uma revisão sistemática sobre o tempo de diagnóstico conduzida baseando-se em 21 estudos do Reino Unido, Holanda, Alemanha, Estados Unidos, Coreia do Sul e China mostrou retardo de diagnóstico estimado em $3,6 \pm 1,9$ meses 30 .

Chama atenção o elevado número de contribuições nas consultas, sobretudo diante do prazo de apenas dez dias para a segunda. Houve 41.787 contribuições, volume que, em prazo reduzido, ultrapassou, em muito, o de outros medicamentos incorporados para doenças raras, como alfataliglicerase (19), idursulfase (625), alfaelosulfase (1.331), galsulfase (2.380) e eculizumabe (2.444). O relatório ainda informa que, desse total, 17.561 eram duplicações de outras, abordavam tema diferente ou estavam em branco, e que 3.793 contribuições traziam rigorosamente o mesmo texto, "indicando uma possivel organização para orientar como fazer a contribuição” 19 (p. 79).

A participação dos diversos atores interessados no processo de avaliação na CONITEC, seja pela inclusão de representações formais, seja pelos mecanismos de consulta e audiência públicas, é certamente um avanço da Lei no 12.401/2011. A inclusão da perspectiva, valores e experiências dos pacientes e usuários pode produzir avaliações mais conectadas com as disposições sociais e contribuir para melhor análise do impacto das tecnologias, ao mesmo tempo em que confere transparência e legitimidade ao processo 31,32. Entretanto, a avaliação, como lócus de decisão para a alocação de recursos, também configura espaço de interesses conflitantes, gerando pressões por parte dos fabricantes, associações de pacientes, médicos, políticos e mídia para o financiamento público de tecnologias que, muitas vezes, carecem de evidências suficientes para sua incorporação e uso racional.

Outro aspecto relacionado a esse processo de incorporação se refere à exigência de acompanhamento dos pacientes em centros de referência, orientado por PCDT, com a coleta e registro de dados clínico-laboratoriais, de modo a permitir a avaliação da efetividade clínica em situação de vida real e reavaliação pela CONITEC no prazo de três anos. Essa novidade já estava presente nas recomendações de incorporação de outros medicamentos nos últimos meses de 2018, como o eculizumabe para hemoglobinúria paroxística noturna 33, e a alfaelusulfase 34 e galsufase 35 para mucopolissacaridose 34,35 .

Esse tipo de modalidade de incorporação condicionada, que no Brasil tem sido denominada uso ad experimentum, vem sendo usada em outros países para pautar a temporalidade da adoção frente ao desenvolvimento de evidências mais robustas de mundo real, que permitam validar as decisões tomadas e informar decisões futuras 36,37. Entretanto, para seu sucesso, é necessário um arcabouço legal, ético e condições estruturais para o monitoramento da efetividade 38 , condições que o Brasil ainda não tem.

Outra inovação, desta vez associada ao processo de compra, é o chamado acordo de partilha de risco. Esse tipo de acordo objetiva contornar o alto grau de incerteza quanto à efetividade de tratamentos e seus altos custos, procurando minimizar o impacto do custo de oportunidade das decisões quanto à incorporação. A vinculação do preço final à avaliação futura é proposta como mecanismo de ajuste da relação preço e valor clínico. Na oportunidade do contrato são fixados potenciais abatimentos no preço teto do medicamento, vinculados a determinados desfechos, aferidos após a celebração do acordo e em reais condições de uso 39.

Países mais desenvolvidos (Espanha, Canadá, Austrália, França, Suécia, Inglaterra) têm utilizado diversas modalidades de incorporação e aquisição ${ }^{40}$. Especificamente em relação à partilha de risco, a cláusula de preço está submetida a evento futuro e incerto; esse risco, elemento que distingue o contrato dos modelos tradicionais de compra e antes assumido integralmente pelo pagador, passa a ser compartilhado com o fornecedor da tecnologia. Apesar de promissor, é considerado um contrato de difícil execução diante dos altos custos de transação e necessária estrutura de avaliação de tecnologias em saúde (ATS) e de coleta de dados para sua execução, entre outros aspectos 1 .

No processo de incorporação do nusinersena no SUS há elementos que aparentemente sugerem não haver partilha de risco. A possibilidade de doação do medicamento aproximaria desse tipo de contrato, uma vez que a doação é uma forma de operacionalizar a flexibilização no preço. Outros 
seriam alguns condicionantes da incorporação, como acompanhamento em centros de referência, a coleta obrigatória de dados e a intenção de reavaliação posterior com base em dados de mundo real 20.

Por outro lado, o relatório final de incorporação não faz mais menção ao fornecimento do medicamento sem custo ${ }^{19}$. Nem a portaria de incorporação nem o relatório de recomendação estabelecem qualquer vínculo de desfecho à fixação do preço. Não há informação sobre abatimento absoluto ou porcentual do preço na ocorrência de determinado evento futuro. Não há como afirmar que incerteza importante esteja presente como item de contrato. Até a redação deste manuscrito, não foi emitida qualquer normativa que estabeleça critérios e regras contratuais para essa modalidade de aquisição no país.

A reavaliação da incorporação no futuro é prerrogativa consagrada da Administração Pública. Além disso, a Diretriz de Investimento e Desinvestimento garante a oportunidade de desincorporação, restrição, retração e substituição da tecnologia, diante de novas evidências quanto à segurança, efetividade, custo e custo-efetividade ou da possibilidade de surgimento de novas e melhores alternativas tecnológicas 41 .

Ressaltamos algumas possíveis consequências dos diferentes aspectos discutidos sobre a institucionalização da ATS em curso desde a promulgação da Lei no 12.410/2011. O estabelecimento de fluxo, prazos e critérios técnico-sanitários e econômicos para a avaliação e incorporação das tecnologias no SUS ajudou a criar regras claras para os atores. Porém, essa institucionalização ainda está em refinamento: medicamentos sem registro sanitário no país foram incorporados 42; avaliações econômicas nem sempre realizadas 43 ; há diferenças nas evidências usadas nas recomendações de incorporação de origem interna e externa 43; e conflitos de interesse também estão presentes e podem ser fatores de influência nas decisões 44 .

As relações próximas entre a CONITEC, com sete representantes de secretarias ministeriais em seu plenário e cuja Secretaria Executiva é exercida pelo Departamento de Gestão e Incorporação de Tecnologias em Saúde, podem trazer problemas para o trabalho e autonomia da Comissão. A literatura internacional sinaliza para instituições independentes, mesmo que financiadas por fundos públicos, como uma estrutura organizacional mais adequada para os órgãos encarregados pela ATS 45.

O estudo conta com algumas limitações. Em primeiro lugar, o SIASG traz compras contratadas e não há garantia sobre registro de gasto de outros entes diferentes da União. É possível, contudo, que grande parte da judicialização do nusinersena tenha sido absorvida pelo Governo Federal, tendo em vista seu altíssimo preço. O relatório final de Recomendação no 400 está ausente da página eletrônica da CONITEC, ainda que o parecer da AGU forneça os detalhes necessários para a compreensão do processo. Por fim, não foram identificados documentos públicos que explicitassem detalhes sobre os contratos de partilha de risco.

\section{Comentários finais}

A CONITEC, institucionalizada como a instância executora de ATS para o SUS, emite relatório final como recomendação para a decisão do secretário da SCTIE/MS e suas recomendações são usualmente subscritas por estes dirigentes. Ainda que seja possível argumentar contra os critérios de priorização do que vem sendo avaliado e incorporado, o processo tem sido conduzido com agilidade e transparência.

A análise da aprovação para a incorporação do nusinersena contraria, em parte, a premissa de transparência do processo. Não houve, aparentemente, novas evidências que justificassem a mudança de recomendação. A demanda da SCTIE/MS à AGU demonstra clara intenção de contornar o processo, tornando a incorporação apenas uma decisão política, sem o suporte essencial da evidência.

As informações quanto à incorporação do nusinersena indicam que não se trata de contrato de partilha de risco. Pressão social, ausência de terapia e alto preço do medicamento são alguns dos critérios para usar esse tipo de acordo. Critérios adicionais incluem incertezas robustas e precisas quanto à efetividade: possibilidade de definir objetivamente desfechos clínicos relevantes, mensuráveis e que tenham correspondência com o preço do medicamento; suficiente estrutura de tecnologia da informação e recursos humanos para a coleta de dados dos pacientes, respeitadas as regras legais 
e éticas; entre outros. Esses acordos servem para ajustar a relação entre valor terapêutico e preço, e recomenda-se que sejam implementados a partir de normativa própria, evitando decisões casuísticas.

Boas práticas de avaliação e incorporação tecnológicas são essenciais para o reconhecimento das decisões pela sociedade, e este reconhecimento se sustenta, entre outros, no atendimento ao disposto nos regimentos legais e na accountability expressa na publicização de cada passo do trabalho. Para ser ferramenta tecnicamente segura, inclusiva, eticamente aceitável e responsável, a ATS precisa estar ancorada em transparência e em critérios de priorização para dar retorno à sociedade sobre decisões que subsidia.

Recomendar a incorporação com evidências em formação, investindo altíssimos valores em um único medicamento, é uma decisão de risco, uma aposta muito grande no futuro da tecnologia, em um país que enfrenta, simultaneamente, judicialização e subfinanciamento da saúde. Medicamentos não podem ser vistos como componentes de "mercados de futuro" ("commodity"). Medicamentos são bens públicos que salvam vidas. Mas todas as vidas são iguais e, portanto, a escolha de quais serão salvas pressupõe critérios muito evidentes de certeza e transparência para a sociedade.

\section{Colaboradores}

Todas as autoras contribuíram com a concepção e planejamento da pesquisa, coleta e análise dos dados, elaboração do texto e figuras e revisão e aprovação da versão final do manuscrito.

\section{Informações adicionais}

ORCID: Rosângela Caetano (0000-0003-14802453); Renata Curi Hauegen (0000-0002-22562966); Claudia Garcia Serpa Osorio-de-Castro (0000-0003-4875-7216).

\section{Agradecimentos}

Ao Departamento de Economia da Saúde, Investimentos e Desenvolvimento, da Secretaria Executiva do Ministério da Saúde, pelo acesso aos dados de compra do medicamento objeto de estudo. Ao Conselho Nacional de Desenvolvimento Científico e Tecnológico (CNPq), processo 304975/2016-8.

\section{Referências}

1. Haugen RC. Risk Sharing Agreements: acordos de partilha de risco e o sistema público de saúde no Brasil - oportunidades e desafios [Tese de Doutorado]. Rio de Janeiro: Instituto de Economia, Universidade Federal do Rio de Janeiro; 2014.

2. Verhaart IEC, Robertson A, Wilson IJ, Aartsma-Rus A, Cameron S, Jones CC, et al. Prevalence, incidence and carrier frequency of 5qlinked spinal muscular atrophy: a literature review. Orphanet J Rare Dis 2017; 12:124.

3. Arnold ES, Fischbeck KH. Spinal muscular atrophy. Handb Clin Neurol 2018; 148:591-601.

4. Darras BT. Spinal muscular atrophies. Pediatr Clin North Am 2015; 62:743-66.

5. Agência Nacional de Vigilância Sanitária. Consulta a medicamentos e hemoderivados. http://portal.anvisa.gov.br/medicamentos/ consultas (acessado em 29/Abr/2019).

6. Brasil. Lei no 12.401, de 28 de abril de 2011. Altera a Lei no 8.080, de 19 de setembro de 1990, para dispor sobre a assistência terapêutica e a incorporação de tecnologia em saúde no âmbito do Sistema Único de Saúde - SUS. Diário Oficial da União 2011; 29 abr.

7. Brasil. Decreto no 7.646, de 21 de dezembro de 2011. Dispõe sobre a Comissão Nacional de Incorporação de Tecnologias no Sistema Único de Saúde e sobre o processo administrativo para incorporação, exclusão e alteração de tecnologias em saúde pelo Sistema Único de Saúde, e dá outras providências. Diário Oficial da União 2011; 22 Dez. 
8. Câmara de Regulação do Mercado de Medicamentos, Agência Nacional de Vigilância Sanitária. Preços máximos de medicamentos por princípio ativo para compras públicas. Tabela atualizada em 21/11/2017. http://portal.anvi sa.gov.br/documents/374947/2829072/LIS TA_CONFORMIDADE_GOV_2017-11-21. pdf/6e53dc1e-fad9-4322-b80a-492db60fb5d8 (acessado em 04/Mai/2019).

9. Câmara de Regulação do Mercado de Medicamentos, Agência Nacional de Vigilância Sanitária. Preços máximos de medicamentos por princípio ativo para compras públicas. Tabela atualizada em 09/04/2018. http://portal. anvisa.gov.br/documents/374947/2829072/ LISTA_CONFORMIDADE_GOV_2018$04-09$.pdf/de 22 aa $75-360 d-4 f 21-9$ c03 2d89a188818b (acessado em 04/Mai/2019).

10. Câmara de Regulação do Mercado de Medicamentos, Agência Nacional de Vigilância Sanitária. Preços máximos de medicamentos por princípio ativo para compras públicas. Tabela atualizada em 15/10/2018. http://portal. anvisa.gov.br/documents/374947/2829072/ LISTA+CONFORMIDADE_2018-10-15.pdf/ a 67c847e-cc06-4c99-9192-b6e1c0b32ee5 (acessado em 04/Mai/2019).

11. Câmara de Regulação do Mercado de Medicamentos, Agência Nacional de Vigilância Sanitária. Preços máximos de medicamentos por princípio ativo para compras públicas. Tabela atualizada de 24/04/2019. http://portal. anvisa.gov.br/documents/374947/2829072/ xls_conformidade_gov_site_2019_04_24. xls/402cfe94-9243-4c0f-a03e-40bccc073f71 (acessado em 04/Mai/2019).

12. Câmara de Regulação do Mercado de Medicamentos, Agência Nacional de Vigilância Sanitária. Preços máximos de medicamentos por princípio ativo para compras públicas. Tabela atualizada de 16/05/2019. http://portal. anvisa.gov.br/documents/374947/2829072/ xls_conformidade_gov_site_2019_05_16.xls/ 0340d96e-0562-4439-8a33-d09af6d91307 (acessado em 20/Mai/2019).

13. Comissão Nacional de Incorporação de Tecnologias no SUS. Nusinersena para o tratamento de pacientes com atrofia muscular espinhal 5q tipo I. Relatório de Recomendação. Agosto de 2018. http://conitec.gov.br/images/ Consultas/Relatorios/2018/Relatrio_nusiner sena_5q_tipo_I_2018_CP.pdf(acessado em 05/ Mai/2019).

14. Comissão Nacional de Incorporação de Tecnologias no SUS. Ata da 69a Reunião da CONITEC. Agosto de 2018. http://conitec.gov. br/images/Reuniao_Conitec/2018/Ata_69Re uniao.pdf (acessado em 04/Mai/2019).

15. Comissão Nacional de Incorporação de Tecnologias no SUS. Ata da 72a Reunião da CONITEC. Novembro de 2018. http://coni tec.gov.br/images/Reuniao_Conitec/2019/ Ata_72Reuniao.pdf (acessado em 04/Mai/ 2019).
16. Comissão Nacional de Incorporação de Tecnologias no SUS. Tecnologias demandadas. http://conitec.gov.br/tecnologias-em-avalia cao\#N (acessado em 05/Mai/2019).

17. Advocacia Geral da União. Parecer no 01377/2018/CONJUR-MS/CGU/AGU. https://www.jota.info/wp-content/ uploads/2019/01/3a943589db4092d821c c1810e19daa2f.pdf (acessado em 24/Abr/ 2019).

18. Comissão Nacional de Incorporação de Tecnologias no SUS. Nusinersena para o tratamento de pacientes com atrofia muscular espinhal 5q. Relatório de Recomendação. Março de 2019. http://conitec.gov.br/images/Con sultas/Relatorios/2019/Relelatorio_Nusiner sena_AME5q_CP_12_2019.pdf (acessado em 05/Mai/2019)

19. Comissão Nacional de Incorporação de Tecnologias no SUS. Nusinersena para o tratamento de pacientes com atrofia muscular espinhal 5q. Relatório de Recomendação no 449. Abril de 2019. http://conitec.gov.br/images/ Relatorios/2019/Relelatorio_Nusinersena_ AME5q_2019.pdf (acessado em 05/Mai/2019).

20. Secretaria de Ciência, Tecnologia e Insumos Estratégicos, Ministério da Saúde. Portaria no 24 , de 24 de abril de 2019. Torna pública a decisão de incorporar o nusinersena para atrofia muscular espinhal (AME) 5q tipo I, no âmbito do Sistema Único de Saúde - SUS. Diário Oficial da União 2019; 25 abr.

21. Figueiredo TA, Osorio-de-Castro CGS, Pepe VLE. Evidence-based process for decisionmaking in the analysis of legal demands for medicines in Brazil. Cad Saúde Pública 2013; 29 Suppl 1:S159-66.

22. Superior Tribunal de Justiça. Recurso Especial: REsp 1657156 RJ 2017/00256297. https://stj.jusbrasil.com.br/jurispruden cia/574252474/recurso-especial-resp1657156-rj-2017-0025629-7 (acessado em 05/ Mai/2019).

23. Santoro Neto L, Lessa F, Nardi EP, Ferraz MB. Stakeholder value judgments in decision-making on the incorporation, financing, and allocation of new health technologies in limited-resource settings: a potential Brazilian approach. Rev Panam Salud Pública 2018; 42:e102.

24. Biogen. Nusinersena para atrofia muscular espinhal 5q. Contribuição para Consulta Pública no 12 de 2019. http://formsus.datasus.gov. br/novoimgarq/46113/9503200_312361.pdf (acessado em 05/Mai/2019).

25. Finkel RS, Mercuri E, Darras BT, Connolly AM, Kuntz NL, Kirschner J, et al. Nusinersen versus sham control in infantile-onset spinal muscular atrophy. N Engl J Med 2017; 377:1723-32.

26. Gerrity MS, Prasad V, Obley AJ. Concerns about the approval of nusinersen sodium by the US Food and Drug Administration. JAMA Intern Med 2018; 178:743-4. 
27. Pinto M, Santos M, Trajman A. Limiar de custo-efetividade: uma necessidade para o Brasil? J Bras Econ Saúde 2016; 8:58-60.

28. De Soarez PC, Novaes HMD. Limiares de custo-efetividade e o Sistema Único de Saúde. Cad Saúde Pública 2017; 33:e00040717.

29. Hyry HI, Stern AD, Cox TM, Roos JC. Limits on use of health economic assessments for rare diseases. QJM 2014; 107:241-5.

30. Lin CW, Kalb SJ, Yeh WS. Delay in diagnosis of spinal muscular atrophy: a systematic literature review. Pediatr Neurol 2015; 53:293-300.

31. Facey K, Boivin A, Gracia J, Hansen HP, Lo Scalzo A, Mossman J, et al. Patients' perspectives in health technology assessment: a route to robust evidence and fair deliberation. Int J Technol Assess Health Care 2010; 26:334-40.

32. Gagnon MP, Desmartis M, Lepage-Savary D, Gagnon J, St-Pierre M, Rhainds M, et al. Introducing patients' and the public's perspectives to health technology assessment: a systematic review of international experiences. Int J Technol Assess Health Care 2011; 27:31-42.

33. Secretaria de Ciência, Tecnologia e Insumos Estratégicos, Ministério da Saúde. Portaria no 77, de 14 de dezembro de 2018. Torna pública a decisão de incorporar o eculizumabe para tratamento de pacientes com hemoglobinúria paroxística noturna (HPN) no âmbito do Sistema Único de Saúde - SUS. Diário Oficial da União 2018; 17 dez.

34. Secretaria de Ciência, Tecnologia e Insumos Estratégicos, Ministério da Saúde. Portaria no 82, de 19 de dezembro de 2018. Torna pública a decisão de incorporar a alfaelosulfase para pacientes com mucopolissacaridose tipo IVa (MPS IVa; síndrome de Morquio A) no âmbito do Sistema Único de Saúde - SUS. Diário Oficial da União 2018; 20 dez.

35. Secretaria de Ciência, Tecnologia e Insumos Estratégicos, Ministério da Saúde. Portaria no 83, de 19 de dezembro de 2018. Torna pública a decisão de incorporar a galsulfase para a terapia de reposição enzimática de longo prazo, em pacientes com diagnóstico confirmado de mucopolissacaridose tipo VI (deficiência de Nacetilgalactosamina 4-sulfa) no âmbito do Sistema Único de Saúde - SUS. Diário Oficial da União 2018; 20 dez.

36. Trueman P, Grainger DL, Downs KE. Coverage with Evidence Development: applications and issues. Int $\mathrm{J}$ Technol Assess Health Care 2010; 26:79-85.
37. Brügger U. A review of Coverage with Evidence Development (CED) in different countries: what works and what doesn't. https:// htai.org/wp-content/uploads/2018/02/CED_ Report_Bruegger_Final_Version.pdf (acessado em 22/Mai/2019).

38. Lexchin J. Coverage with evidence development for pharmaceuticals: a policy in evolution? Int J Health Serv 2011; 41:337-54.

39. Adamski J, Godman B, Ofierska-Sujkowska G, Osińska B, Herholz H, Wendykowska K, et al. Risk sharing arrangements for pharmaceuticals: potential considerations and recommendations for European payers. BMC Health Serv Res 2010; 10:153.

40. Carlson JJ, Gries KS, Yeung K, Sullivan SD, Garrison Jr. LP. Current status and trends in performance-based risk-sharing arrangements between healthcare payers and medical product manufacturers. Appl Health Econ Health Policy 2014; 12:231-8.

41. Ministério da Saúde. Diretrizes metodológicas: avaliação de desempenho de tecnologias em saúde. http://conitec.gov.br/images/Artigos_ Publicacoes/Diretrizes/DIRETRIZ_AdTS_fi nal_ISBN.pdf (acessado em 22/Mai/2019).

42. Pedro EM, Caetano R, Teodoro CRS, Steffen RE, Silva RM. Incorporação de medicamentos sem registro sanitário no SUS: um estudo das recomendações da Comissão Nacional de Incorporação de Tecnologias no período 2012 2016. Vigil Sanit Debate 2018; 6:12-21.

43. Yuba TY, Novaes HMD, De Soárez PC. Challenges to decision-making processes in the national HTA agency in Brazil: operational procedures, evidence use and recommendations. Health Res Policy Syst 2018; 16:40.

44. Souza KA, Souza LEPF. Incorporação de tecnologias no Sistema Único de Saúde: as racionalidades do processo de decisão da Comissão Nacional de Incorporação de Tecnologias no Sistema Único de Saúde. Saúde Debate 2018; 42(n. esp. 2):48-60.

45. Stephens JM, Handke B, Doshi JA. International survey of methods used in health technology assessment (HTA): does practice meet the principles proposed for good research? Comp Eff Res 2012; 2:29-44. 


\section{Abstract}

In April 2019, a ruling was signed for the incorporation of the drug nusinersen by the Brazilian Unified National Health System (SUS). Nusinersen is the most expensive drug ever incorporated by the SUS and is used to treat type I $5 q$ spinal muscular atrophy. The incorporation has been described as a milestone in decision-making on new technologies in the SUS, enabled through a risk-sharing agreement. The article discusses the process involved in the incorporation of nusinersen, highlighting the context, timing, and technical issues, in addition to possible consequences for the institutionalization of health technology assessment (HTA) in the SUS. The study used an exploratory method, reviewing public information produced by the Commission for Incorporation of Technologies in the SUS (CONITEC) and searches in government databanks on prices and purchases. A timeline was produced, describing the key points in the process of incorporation. There were two formal requests for the drug's incorporation. The first was submitted by the Division of Science, Technology, and Strategic Inputs (SCTIE) of the Brazilian Ministry of Health and was turned down unanimously in November 2018. This was followed by a petition by the head of the SCTIE to the Attorney General's Office (AGU) to overrule the recommendation by the CONITEC plenary. The AGU recommended a new submission, made by the drug's manufacturing company, which was approved unanimously in March 2019. The was no addition of new evidence or a price reduction to justify the change of decision. No elements were identified in the risk-sharing agreement. This suggests problems of transparency and accountability, as well as risks in the process of institutionalization of HTA that had been underway in the SUS.

Health Technology Assessment; Orphan Drug; Financial Risk Sharing; Unified Health System

\section{Resumen}

En abril de 2019, se firmó el decreto de incorporación del medicamento nusinersén en el Sistema Único de Salud brasileño (SUS). Es el medicamento más caro que se ha incorporado al SUS para su uso en el tratamiento de la atrofia muscular espinal 5q tipo I. La incorporación del mismo está considerada como un marco de referencia en la toma de decisiones sobre nuevas tecnologías en el SUS, que puede ser viable mediante el acuerdo de distribución de riesgo. El trabajo discute el proceso de incorporación del nusinersén, destacando aspectos contextuales, temporales y técnicos, además de posibles consecuencias para la institucionalización de la evaluación de tecnologías en salud (ETS) en el SUS. El trabajo siguió el método exploratorio, con una revisión de la información pública, generada por la Comisión de Incorporación de Tecnologías en el SUS (CONITEC) y la búsqueda en bancos de datos gubernamentales de precios y compras. Se creó una línea temporal, describiendo los puntos-clave del proceso de incorporación. Hubo dos peticiones de incorporación del medicamento. La primera, sometida a la Secretaría de Ciencia, Tecnología e Insumos Estratégicos (SCTIE) del Ministerio de Salud, rechazada por unanimidad, en noviembre de 2018. A lo que le siguió la petición del Secretario de la SCTIE a la Abogacía-General de la Unión (AGU), para que pudiese decidir en otro sentido respecto a la recomendación del pleno de la CONITEC. La AGU recomendó una nueva remisión, realizada por la empresa productora y aprobada por unanimidad, en marzo de 2019. No se produjo un incremento de nuevas evidencias o una reducción del precio que justificasen el cambio de decisión. No se identificaron los elementos constituyentes del acuerdo de distribución de riesgo. Se señalaron los problemas de transparencia y rendición de cuentas, así como riesgos para el proceso de institucionalización de la ETS que estaba en curso en el SUS.

Evaluación de Tecnologías de Salud;

Medicamento Huérfano; Prorrateo de Riesgo

Financiero; Sistema Único de Salud
Recebido em 27/Mai/2019

Versão final reapresentada em 17/Jul/2019

Aprovado em 23/Jul/2019 